\title{
Colorimetric Detection Based on Localized Surface Plasmon Resonance for Determination of Chemicals in Urine
}

\author{
Chutiparn LertvachiraPaiboon, ${ }^{\dagger}$ Akira Baba,${ }^{\dagger}$ Kazunari Shinbo, and Keizo Kato \\ Graduate School of Science and Technology, Niigata University, 8050 Ikarashi 2-nocho, Nishi, Niigata 950-2181, \\ Japan
}

\begin{abstract}
Colorimetric sensors based on localized surface plasmon resonance (LSPR) have attracted much attention for biosensor and chemical sensor applications. The unique optical effect of LSPR is based on the nanostructure of noble metals (e.g., $\mathrm{Au}, \mathrm{Ag}$, and $\mathrm{Al}$ ) and the refractive index of the environment surrounding these metal nanomaterials. When either the structure or the environment of these nanomaterials is changed, their optical properties change and can be observed by spectroscopic techniques or the naked eye. Colorimetric-probe-based LSPR provides a simple, rapid, real-time, nonlabelled, sensitive biochemical detection and can be used for point-of-care testing as well as rapid screening for the diagnosis of various diseases. Gold and silver nanoparticles, which are the two most widely used plasmonic nanomaterials, demonstrate strong and sensitive LSPR signals that can be used for the selective detection of several chemicals in biochemical compounds provided by the human body (e.g., urine and blood). This information can be used for the diagnosis of several human health conditions. This paper provides information regarding colorimetric probes based on LSPR for the detection of three major chemicals in human urine: creatinine, albumin, and glucose. In addition, the mechanisms of selective detection and quantitative analysis of these chemicals using metal nanoparticles are discussed along with colorimetric-detection-based LSPR for many other specific chemicals that can be detected in urine, such as catecholamine neurotransmitters, thymine, and various medicines. Furthermore, issues regarding the use of portable platforms for health monitoring with colorimetric detection based on LSPR are discussed.
\end{abstract}

Keywords Localized surface plasmon resonance, metal nanoparticles, urine, creatinine, albumin, glucose

(Received September 20, 2020; Accepted October 24, 2020; Advance Publication Released Online by J-STAGE October 30, 2020)

1 Introduction

2 Chemical Diagnosis in Urine Using Plasmonic

Nanomaterials

$2 \cdot 1$ Creatinine

$2 \cdot 2$ Albumin

$2 \cdot 3$ Glucose
930

930
2.4 Other chemicals in urine

3 Portable Platforms Based on Colorimetric Probes Using LSPR for Human Health Monitoring 937

4 Conclusions

5 Acknowledgements

6 References
937

939

939

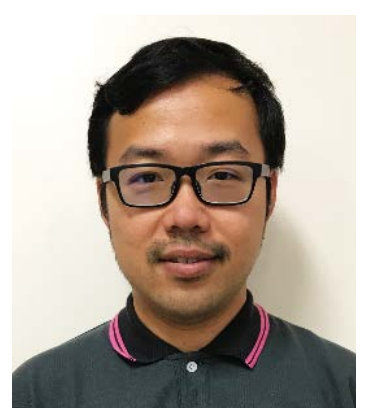

Chutiparn LERTVACHIRAPAIBOON obtained his B.Sc. degree in Chemistry from King Mongkut's University of Technology Thonburi, Thailand and M.Sc. degree in Chemistry from Chulalongkorn University, Thailand. He received his Ph.D. in Science at Chulalongkorn University, Thailand and Ph.D. in Electrical and Electronic Engineering at Niigata University, Japan in 2015 (Double Degree). In 2016, he joined in Max-VolmerLaboratorium, Technische Universität Berlin, Germany as an Alexander von Humboldt Research Fellow. Since 2015, he has been with Niigata University, Japan, where he is now Assistant Professor of the Graduate School of Science and Technology. His research interests include synthesis and characterization of plasmonic nanoparticles and sensor applications.

$\dagger$ To whom correspondence should be addressed. E-mail: chutiparn.1@eng.niigata-u.ac.jp (C. L.); ababa@eng.niigata-u. ac.jp (A. B.)

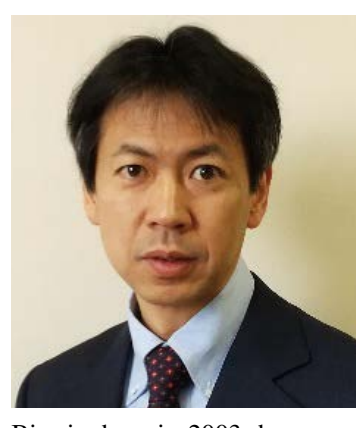

Akira BABA is a Professor at Graduate School of Science and Technology and Faculty of Engineering, Niigata University, Japan. He received his Ph.D. in Electrical and Electronic Engineering at Niigata University, Japan in 1999. In 1999, he joined the Max-Planck-Institute for Polymer Research, Mainz, Germany as an Alexander von Humboldt Research Fellow. Then, he worked at National University of Singapore in the Department of Materials Science for a half year. After a short stay at University of Alabama at Birmingham in 2003, he moved to the University of Houston, Department of Chemistry where he has worked as Research Associate Staff. He has been with Niigata University since 2007. His research interests involve developments of surface sensitive techniques, nanofabrication and analysis of organic thin films/biomolecules, and their optoelectronic applications of versatile and novel chemical sensing systems using stimuli-sensitive polymers and colloidal gold for biological analysis. 


\section{Introduction}

Localized surface plasmon resonance (LSPR) is a unique optical property of noble-metal nanostructures (e.g., $\mathrm{Au}, \mathrm{Ag}$, and $\mathrm{Al}$ ) based on the coupling between an external electromagnetic field and a collective electron oscillation in the conduction band of the nanostructures. ${ }^{1-7}$ The resulting local electric field is very sensitive to structural changes (size and shape) in metal nanostructures as well as the refractive index of the environment, leading to detectable changes in their optical properties. ${ }^{1-7}$ Gold nanoparticles (AuNPs) and silver nanoparticles (AgNPs) have unique optical characteristics in the visible-light region, which enables the monitoring of their LSPR properties by ultravioletvisible (UV-vis) spectrometry and cameras, as well as the naked eye. 1,2,7-11 Colorimetric detection, benefiting from the LSPR phenomenon of these plasmonic nanomaterials, is used for several biosensor applications, including the detection of chemical compounds in the human body. ${ }^{7-11}$ Urine is a liquid byproduct of the metabolism of the human body, extracted by the kidneys as a waste solution from the bloodstream along with excess products, such as water and sugar. Three of the main chemicals often found in urine, namely creatinine, albumin, and glucose, can be detected by the colorimetric method based on LSPR. This paper discusses the most successful examples of detection of these three chemicals from human urine. Urine detection using LSPR has many advantages. For example, the LSPR technique is a colorimetric detection that allows for the observation of color change by the naked eye and quantification by UV-vis spectroscopy. Furthermore, when using a colorimetric probe for creatinine detection with plasmonic nanomaterials, there is no need to use explosive picric acid, as is required in the Jaffe-based method. We are interested in the three aforementioned chemicals in urine because their presence is directly related to human health conditions. Even though the detection of these chemicals in urine samples cannot alone provide exact diagnosis results (unlike in the case of blood samples), it can provide useful information regarding point-ofcare (POC) testing and rapid screening for the disease diagnosis. Moreover, this platform is cheap, making it useful for the routine monitoring of human health. This paper also discusses several other specific chemicals that can be detected from human urine and colorimetric detection based on the LSPR technique, such as catecholamine neurotransmitters, thymine, and various medicines. Recently, portable platforms based on colorimetric probes using LSPR have attracted much attention for biosensor applications, including urine detection. These platforms are also discussed in this paper.

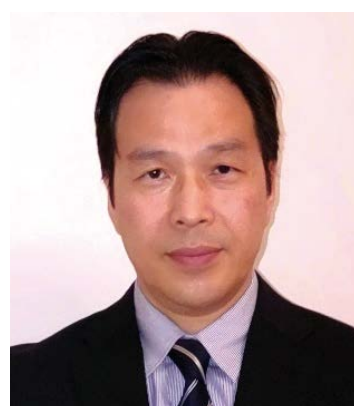

Kazunari SHINBo received the B.E. M.E. and D.E. degrees from Niigata University, Niigata, Japan in 1990, 1992 and 1995, respectively. Since 1995, he has been with Niigata University where he is now a Professor in the Faculty of Engineering. His current research interests are in property of organic ultrathin films and its applications. Dr. Shinbo is a member of the Institute of Electrical Engineers of Japan and the Japan Society of Applied Physics.

\section{Chemical Diagnosis in Urine Using Plasmonic Nanomaterials}

\subsection{Creatinine}

Creatinine is one of the chemicals found in human serum and urine. It is a final product and a chemical waste molecule in the body, because it has no useful biological function. Creatinine is filtered by the kidneys and excreted in urine. The normal concentration of creatinine in serum and the regular amount of its excretion through urine are $45-140 \mu \mathrm{M}$ and $0.8-2.0 \mathrm{~g}$ per day, respectively. ${ }^{12}$ Men have slightly higher levels of creatinine than women due to their higher muscle mass. The determination of creatinine levels in biological fluids is important for evaluating renal dysfunction, thyroid malfunction, and muscle damage. ${ }^{13-15}$ A creatinine level lower than $45 \mu \mathrm{M}$ indicates decreased muscle weight, which is a sign of muscular disorder. ${ }^{15}$ Meanwhile, a creatinine level above $140 \mu \mathrm{M}$ is a sign of renal kidney dysfunction. ${ }^{12,16}$ The measure of the urinary creatinine level is also essential for clinical assays of renal function. The normal creatinine level for healthy humans ranges from approximately 4.4 - 18.0 mM. ${ }^{14,17,18}$ A level outside this range requires further diagnosis to identify the type of abnormality. ${ }^{14,17,18}$ Nonetheless, a major advantage of using urine versus blood samples is the simple sampling method, which generally confers no disturbance to patient integrity.

Several methods, so far, have been proposed for creatinine analysis, including electrochemical detection, spectroscopy, and enzymatic methods coupled with chromatographic techniques. ${ }^{19-22}$ Colorimetric methods using the LSPR of metal nanoparticles (MNPs, i.e., AuNPs and AgNPs) have attracted much attention due to the unique optical properties of these materials, which can be easily observed by a UV-vis spectrometer or the naked eye, while the metal surface can be modified for the selective detection of specific molecules..$^{23-28}$ Since AuNPs and AgNPs are the two most commonly used materials for this method, we focus on their use in creatinine detection. Here, the aggregation mechanisms of these plasmonic materials in the presence of creatinine are discussed in detail, with aggregation being their main principle.

\section{2·1·1 Gold nanoparticles}

The use of citrate-capped AuNPs as a colorimetric probe has been demonstrated as a selective and sensitive method for determining creatinine levels. ${ }^{23}$ The detection range of this system, which covers creatinine concentrations in the range of $0.1-20.0 \mathrm{mM}$, was previously obtained as $80 \mu \mathrm{M}$ in buffer solutions. The color of AuNP colloidal solutions changed from red to blue and the LSPR peak of the AuNPs shifted from $520-670 \mathrm{~nm}$ due to AuNP aggregation in the presence of

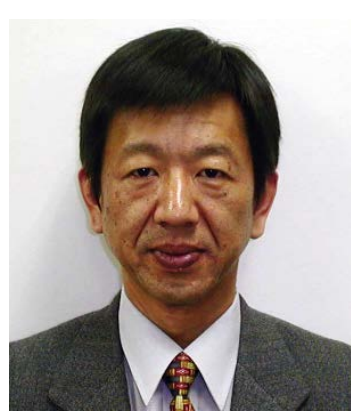

Keizo Kato received the B.E., M.E. and Dr. Eng. Degrees in Electrical and Electronic Engineering from Tokyo Institute of Technology, Japan in 1982, 1984, 1987, respectively. Since 1987, he has been with Niigata University, Japan, where he is now a Professor of the Graduate School of Science and Technology. During $1999-2000$, he was a Visiting Professor of The University of Sheffield, UK. The areas of his research involve electrical and electronic materials, thin films and organic electronics. Recently his research focuses on evaluation and device applications of nanostructured organic thin films utilizing surface plasmon excitations. Dr. Kato is a Fellow of the Institute of Electrical Engineers of Japan, a Fellow of the Institute of Electronics, Information and Communication Engineers, Japan, and a member of IEEE and the Japan Society of Applied physics. 
(a)

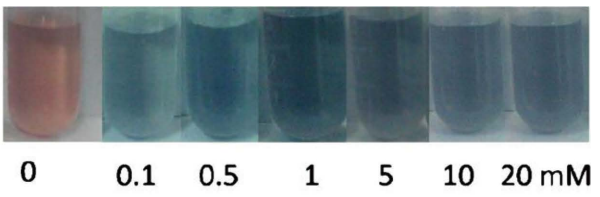

(b)
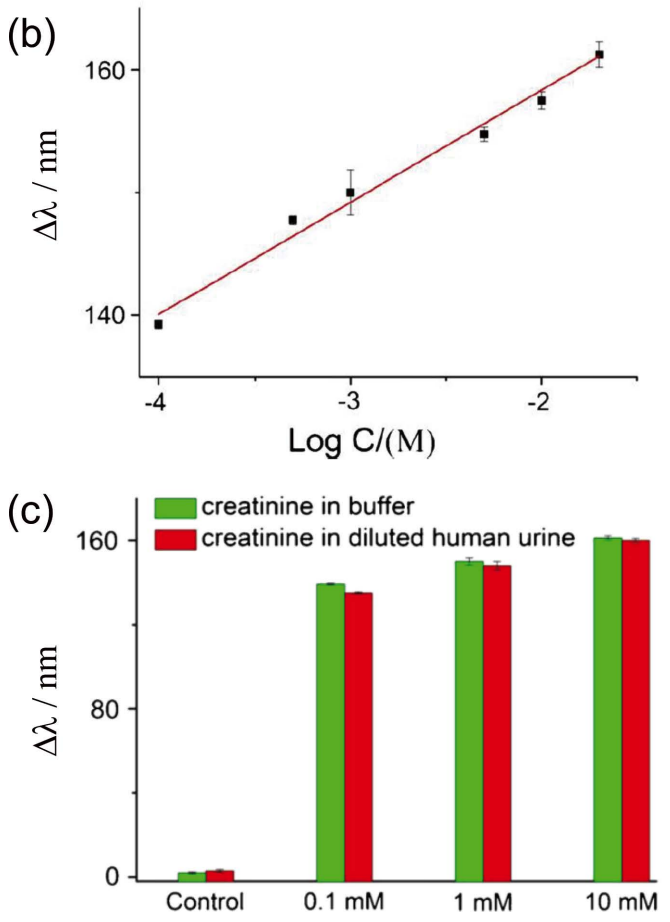

Fig. 1 (a) Photographic images and (b) calibration curve of AuNP solution in the presence of $0.1-20 \mathrm{mM}$ creatinine. (c) Histograms of the LSPR peak shifts of AuNPs in the presence of different creatinine concentrations in buffer (green columns) and diluted urine (red columns) solutions. Copyright $\odot 2015$ Springer-Verlag Wien. ${ }^{23}$

creatinine. A photographic image and calibration curve of creatinine detection in this concentration range are shown in Figs. 1(a) and 1(b), respectively. These citrate-capped AuNPs were particularly used for human urine detection. The response of a AuNP colloidal solution to various creatinine concentrations in buffer, along with the diluted urine solutions, is shown in Fig. 1(c). This assay also showed a good selectivity over interfering substances including various inorganic ions, small organic compounds, proteins, and biothiols. The results indicated that citrate-capped AuNPs can be successfully used for urinary creatinine detection. This method demonstrated good recovery rates of $97-102 \%$.

Meanwhile, several techniques have been developed to obtain highly accurate values of urinary creatinine without any interference from the other components in urine. ${ }^{24-26}$ In one technique, AuNPs were used for detecting creatinine in human urine after its extraction using sulfonic acid-functionalized silica gel. $^{24}$ A schematic of this extraction method is shown in Fig. 2(a). The removal of the interfering effects of the urine matrix on creatinine detection by AuNPs was achieved by selectively inducing the adsorption of creatinine on the functionalized silica gel. The adsorbed creatinine was then eluted using a $\mathrm{NaCl}$ solution. After separating the solid, the supernatant was used for creatinine analysis by AuNPs. This technique could determine creatinine concentrations ranging from 15 - $40 \mathrm{mg} \mathrm{L}{ }^{-1}$, with a detection limit of $13.7 \mathrm{mg} \mathrm{L}^{-1}$. The (a)

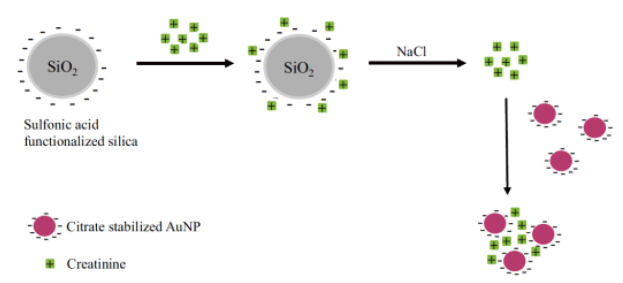

(b)

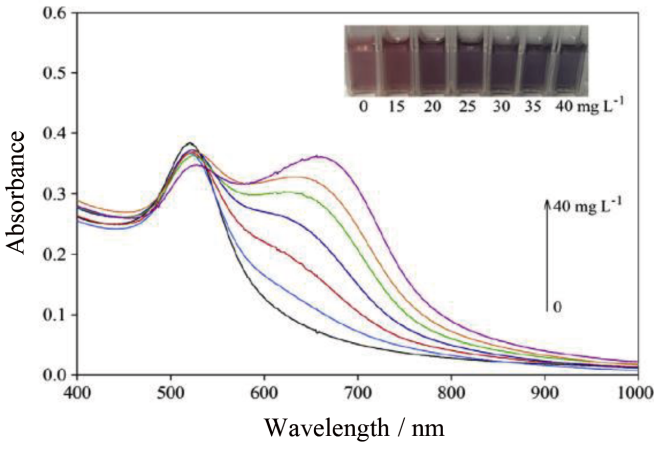

(c)

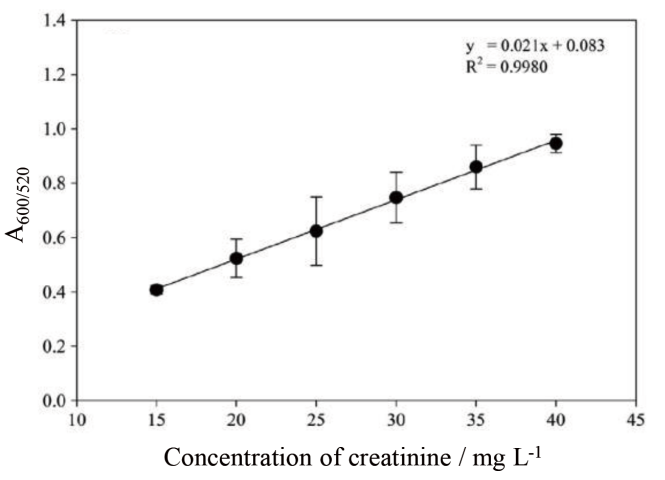

Fig. 2 (a) Proposed mechanism of creatinine extraction and reaction with AuNPs. (b) UV-vis absorption spectra of AuNPs in the presence of creatinine extracted from different standard solutions and (c) the calibration curve for creatinine determination. Copyright $\odot 2018$ Elsevier B.V.24

UV-vis absorption spectra and calibration curve of AuNPs in the presence of creatinine extracted from different standard solutions are shown in Figs. 2(b) and 2(c), respectively. This method achieved recovery rates of $96.1-104.5 \%$ for the detection of urinary creatinine.

A coordination chemistry-based AuNP platform has been used for creatinine determination. ${ }^{25}$ Du et al. developed a uric acidand $\mathrm{Hg}^{2+}$-modified AuNP system for the selective detection of creatinine in a complex matrix. In AuNP solutions, the assay revealed an obvious color change from red to blue in relation to the creatinine concentration. Under the optimum conditions, the detection limit of this method reached $1.6 \times 10^{-9} \mathrm{M}$. For the urinary creatinine samples, suitable recovery rates in the range of $94.0-103.4 \%$ were obtained. Later, a mercury-free coordination chemistry-based AuNP platform was developed by the same research group. ${ }^{26}$ In addition, a sensor comprising adenosine $/ \mathrm{Ag}^{+}$-AuNPs was developed for selectively identifying creatinine. Typical LSPR spectra of functionalized AuNPs with different concentrations of creatinine, as well as the linear relations between the LSPR intensities at a ratio of $A_{630 \mathrm{~nm} / 520 \mathrm{~nm}}$ and creatinine concentrations covering a range of $0.2-1.4 \mu \mathrm{M}$ in buffer solution and $0.2-2.0 \mu \mathrm{M}$ in artificial urine, are shown in Fig. 3. The coordination chemistry-based AuNP platforms exhibited good selectivity, sensitivity, and obvious color changes in the determination of creatinine. 
(a)
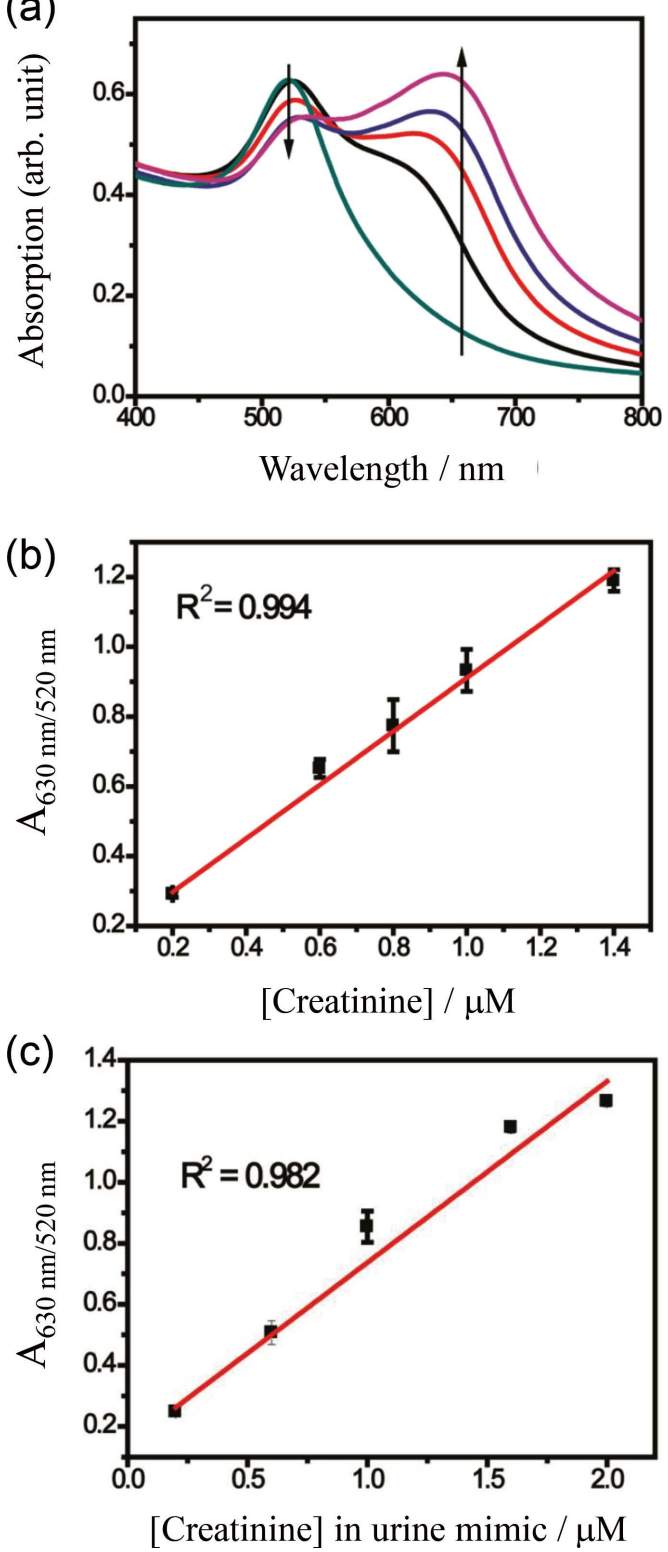

Fig. 3 (a) UV-vis spectra of adenosine/ $\mathrm{Ag}^{+}-\mathrm{AuNP}$ in the presence of different creatinine concentrations in buffer solution. Plots of the

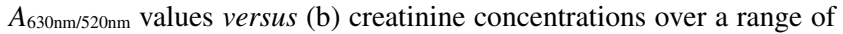
$0.2-1.4 \mu \mathrm{M}$ in buffer solution and (c) $0.2-2.0 \mu \mathrm{M}$ in artificial urine. Copyright $@ 2016$ American Chemical Society. ${ }^{26}$

\subsubsection{Silver nanoparticles}

Colloidal solutions of AgNPs have also been used for creatinine detection. ${ }^{27,28}$ Mohammadi and Khayatian prepared 2,2-thiodiacetic acid-capped AgNPs for determining the creatinine concentration. ${ }^{27}$ Creatinine induced the aggregation of AgNPs capped with 2,2-thiodiacetic acid, while other biomolecules did not show any effect. The color of the AgNP solution turned green in the presence of creatinine, whose concentration was quantified by UV-vis spectroscopy. This colorimetric probe allowed rapid quantification of creatinine in the concentration range of $0.01-1.0 \mu \mathrm{M}$, with a detection limit of $3.0 \mathrm{nM}$. The selectivity of this method was also evaluated. A good selectivity over interfering substances including cations, amino acids, and some of other related chemicals in urine was exhibited. This method was applied to the determination of creatinine in urine and serum samples. The recovery rate for (a)
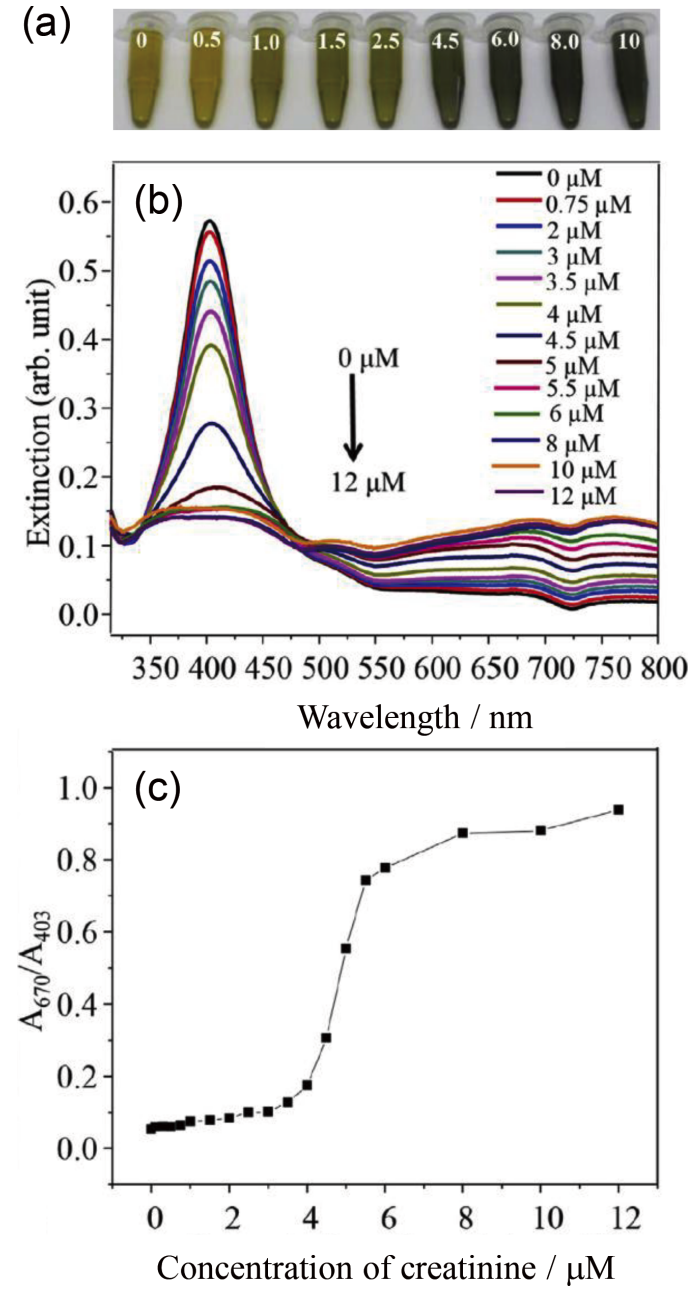

Fig. 4 (a) Photographic image of citrate-capped AgNP colloidal solutions mixed with different concentrations of creatinine at $\mathrm{pH} 12$ with colorimetric changes visible up to $10 \mu \mathrm{M}$ creatinine concentration. (b) LSPR spectra of citrate-capped AgNP colloidal solutions in the presence of different creatinine concentrations ranging $0-12 \mu \mathrm{M}$. (c) Plots depicting the relation between the $A_{670 \mathrm{~nm} / 403 \mathrm{~nm}}$ extinction ratio of the citrate-capped AgNP colloids and creatinine concentration at pH 12. Copyright@2018 Elsevier B.V. ${ }^{28}$

urinary creatinine detection using these AgNPs ranged 89.4 $102.1 \%$.

Citrate-capped AgNPs have also been used for detecting creatinine in urine under alkaline conditions. ${ }^{28}$ The citratecapped AgNPs showed a linear correlation between the $A_{670 \mathrm{~nm} / 403 \mathrm{~nm}}$ extinction ratio and creatinine concentration in the range of $0-4.2 \mu \mathrm{M}$, with a detection limit of $53.4 \mathrm{nM}$. This approach required no additional surface modification of AgNPs to simplify the synthesis method or detection. A photographic image of AgNP colloidal solutions mixed with different concentrations of creatinine, their typical LSPR spectra, and the plots depicting the relation between the $A_{670 \mathrm{~nm} / 403 \mathrm{~nm}}$ extinction ratio of AgNPs and creatinine concentrations at $\mathrm{pH} 12$ are shown in Fig. 4. The detailed mechanisms involved in creatinine detection using AuNPs and AgNPs are outlined in the next section.

2.1.3 Principle of colorimetric methods for creatinine detection based on AuNPs and AgNPs

This paper focuses on the interaction between functionalized MNPs and creatinine, since both AuNPs and AgNPs are the 
(a)

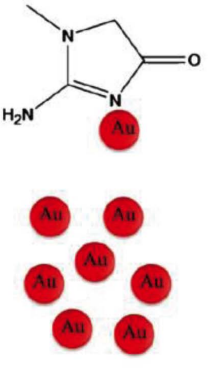

Citrate -stabilized AuNPs
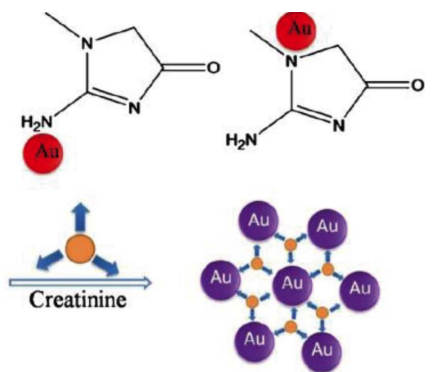

Cross-linked AuNPs (b)

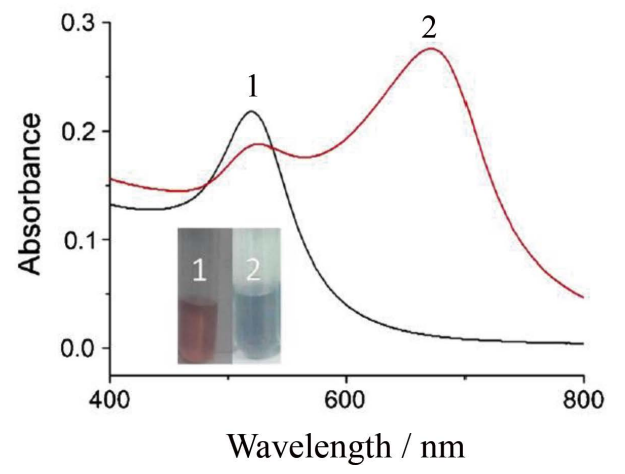

Fig. 5 (a) Schematic of the proposed crosslinking reaction mechanism of creatinine and AuNPs caused by a color change in colloidal AuNP solution from red to blue. (b) UV-vis spectra of AuNP colloidal solution in (1) the absence and (2) presence of $1 \mathrm{mM}$ creatinine ( $\mathrm{pH}$ 6.0). The inset shows the photographic images of the corresponding systems. Copyright $\odot 2015$ Springer-Verlag Wien. ${ }^{23}$

most widely used materials for creatinine detection. Creatinine has two tautomer forms and the preferred aqueous tautomer contains three nitrogen-containing groups (Fig. 5(a)). ${ }^{29}$ The surfaces of citrate-stabilized AuNPs are capped by negatively charged citrate ions, which are stabilized against aggregation through the electrostatic repulsion among the nanoparticles. Nevertheless, the interaction between AuNPs and citrate is weak. It has been demonstrated that citrate-capped AuNPs can rapidly bind electron-rich nitrogen-containing compounds, such as melamine and luminol, through a coordination interaction between $\mathrm{N}$ atoms and AuNPs..$^{30,31}$ Primary amines were used to modify the faces of AuNPs. ${ }^{32}$ Nitrogen in an aromatic ring also exhibited strong binding affinity to AuNPs. ${ }^{33}$ Therefore, when creatinine with three binding sites was added to citrated-capped AuNPs, the ligand-exchange reaction of creatinine and citrate ions first occurred, decreasing the number of citrate ions on the AuNP surface, and finally, crosslinking them. ${ }^{23}$ When the crosslinking reaction induced AuNP aggregation, the decrease in the LSPR of the AuNPs at $520 \mathrm{~nm}$ and an aggregated AuNP peak at approximately $670 \mathrm{~nm}$ were observed. The LSPR spectra and corresponding photographic images of AuNP solutions both in the presence and absence of creatinine are shown in Fig. 5(b).

Coordination chemistry is a widely used strategy in the colorimetric detection of specific targets based on a AuNP platform. ${ }^{25,26}$ Adenosine/ $\mathrm{Ag}^{+}$complexes on the AuNP surface can functionally recognize creatinine, as it can form complexes with $\mathrm{Ag}^{+}$in an aqueous solution. ${ }^{26,34,35}$ Adenosine/ $\mathrm{Ag}^{+}$-AuNPs demonstrates an excellent selectivity to creatinine among various ions and biomolecules, and can be used for urinary creatinine detection. ${ }^{26}$ The $\mathrm{m} / \mathrm{z}$ signals for creatinine/ $/ \mathrm{Ag}^{+}$,

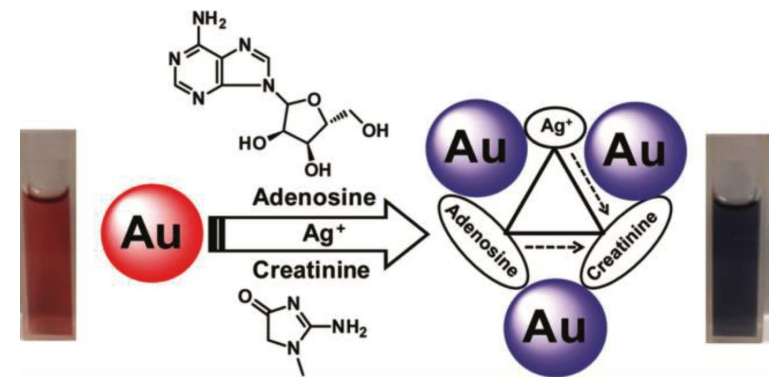

Fig. 6 Schematic of the colorimetric assay of creatinine based on AuNPs through the synergistic coordination chemistry of creatinine with adenosine and $\mathrm{Ag}^{+}$. Copyright $\odot 2016$ American Chemical Society. ${ }^{26}$

adenosine $/ \mathrm{Ag}^{+}$, and adenosine/creatinine/ $\mathrm{Ag}^{+}$complexes have also been observed according to the mass spectroscopy results obtained from adenosine/ $\mathrm{Ag}^{+}-\mathrm{AuNPs}$ in the presence of creatinine. These have proved the formation of an adenosine/ creatinine $/ \mathrm{Ag}^{+}$complex on the AuNP surfaces. Furthermore, it has been observed that AuNP aggregation due to complex formation directly depends on the creatinine concentration. In the presence of creatinine, a decrease in LSPR intensity at $520 \mathrm{~nm}$ was observed along with a new peak at approximately $630 \mathrm{~nm}$. Moreover, a red-to-blue color change was visually observable within 1 min (photographic image shown in Fig. 6). A schematic of the colorimetric assay of creatinine based on AuNPs through the synergistic coordination chemistry of creatinine with adenosine and $\mathrm{Ag}^{+}$is shown in Fig. 6 .

In the presence of creatinine, citrate-capped AuNPs can themselves aggregate, whereas AgNPs require alkaline conditions to drive the reaction. ${ }^{28}$ Alula et al. investigated the effect of $\mathrm{pH}$ on the aggregation of citrate-capped AgNPs in the presence of creatinine. Three distinct mixtures of AgNPs containing (a) $\mathrm{NaOH}$, (b) creatinine, and (c) a mixed solution of creatinine and $\mathrm{NaOH}$ were prepared. Changes in color and LSPR spectrum were observed only in the mixed solution containing creatinine and $\mathrm{NaOH}$ (c). The color of the AgNP colloidal solution under this condition changed from yellow to dark blue, and a decrease in the LSPR peak at $403 \mathrm{~nm}$ was observed (Fig. 7). These results indicate that alkaline conditions are necessary to drive the interaction between creatinine and citrate-capped AgNPs. The decrease in the LSPR peak at $403 \mathrm{~nm}$ represented a decrease in the number of individual AgNPs in the colloidal solution. Furthermore, the observable peak at approximately $670 \mathrm{~nm}$ under condition (c) represented an aggregation of AgNPs. The degree of color change of AgNPs and a decrease in LSPR intensity at $403 \mathrm{~nm}$ depended on the concentration of creatinine. The aggregation of AgNPs in the presence of creatinine under alkaline conditions can be explained by the following mechanism. As two carboxylate groups of citrate molecules are bound to the AgNP surface, only one remaining carboxylate group is available to contribute to the negative surface charge of the AgNPs. Citrate-capped AgNPs maintain their stability under alkaline conditions because that carboxylate group sustains the electrostatic repulsion between the individual AgNPs. ${ }^{36,37}$ The amino tautomer is a thermodynamically stable form of creatinine, even under alkaline conditions. ${ }^{38}$ Thus, hydrogen bonds are exposed to carboxylate groups of citrate, and an intermolecular hydrogen bonding network is formed between surface-bound creatinine molecules that effectively crosslink the citrate-capped AgNPs, leading to aggregation. ${ }^{28}$

MNPs can be used not only for colorimetric detection, but 


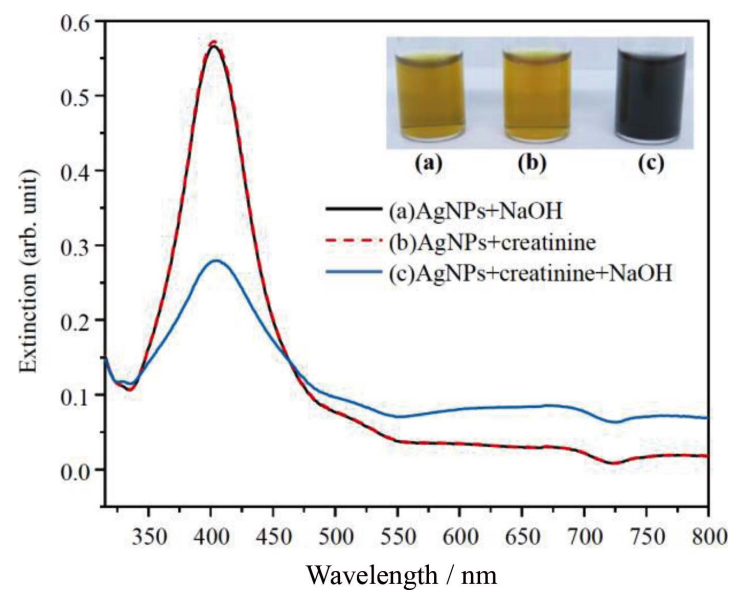

Fig. 7 LSPR spectra of citrate-capped AgNP colloids mixed with (a) $\mathrm{NaOH}$, (b) creatinine, and (c) a mixed solution of creatinine and $\mathrm{NaOH}$. The inset shows photographic images corresponding to these conditions. Copyright $\odot 2018$ Elsevier B.V. ${ }^{28}$

also in several other ways. The following is an outline of the other techniques in which MNPs, especially AuNPs, have been used for creatinine detection: dynamic light scattering (DLS) and surface-enhanced Raman spectroscopy (SERS). ${ }^{39,40}$ A sensitive and selective approach for creatinine detection using calix[4]arene-functionalized AuNPs detected by DLS has been developed. ${ }^{39}$ The fast response of this material to creatinine is attributed to hydrogen bonding and the electrostatic interaction between the amine group of creatinine and the calix[4]arene compound, which can be used to quantify the creatinine concentration. The SERS technique has been used for detecting several chemicals in urine, including creatinine, albumin, and urea. ${ }^{40}$ To achieve this, AuNPs were trapped in the nanochannels of a Blu-ray digital versatile disc (BRDVD), a structure that demonstrates stable and reproducible SERS signals. In case of creatinine, the minimum concentration that could be detected using this technique was $0.2 \mu \mathrm{g} \mathrm{mL}^{-1}$, while the minimum concentrations of albumin and urea were 0.1 and $0.6 \mu \mathrm{g} \mathrm{mL}^{-1}$, respectively. As the SERS spectra obtained from this substrate were stable and the minimum concentrations of the tested chemicals were lower than the normal range detected in human urine, the SERS technique using this substrate can very likely be used for clinical purposes.

Colorimetric detection using paper-based platforms and fluorometric probes has also led to several attractive and practical methods for creatinine detection. ${ }^{41,42}$ The recovery of creatinine from spiked samples obtained using this technique was found to have an excellent range. These colorimetric platforms also provide a simple and rapid detection of creatinine with a low consumption of reagents. Nevertheless, the colorimetric approach using the LSPR of plasmonic materials reveals obvious changes in color that can be observed by the naked eye and quantified by a UV-vis spectrometer, making it even more attractive.

\subsection{Albumin}

Normally, urinary protein is formed of albumin, which is a soluble protein, and constitutes about one half of the blood serum protein. The albumin present in urine is abnormal, as it should be retained in the bloodstream by the kidneys. Albumin may be detected in urine but the concentration should be lower than $0.3 \mathrm{mg} \mathrm{dL}^{-1} \cdot{ }^{43,44}$ High concentrations of albumin in urine
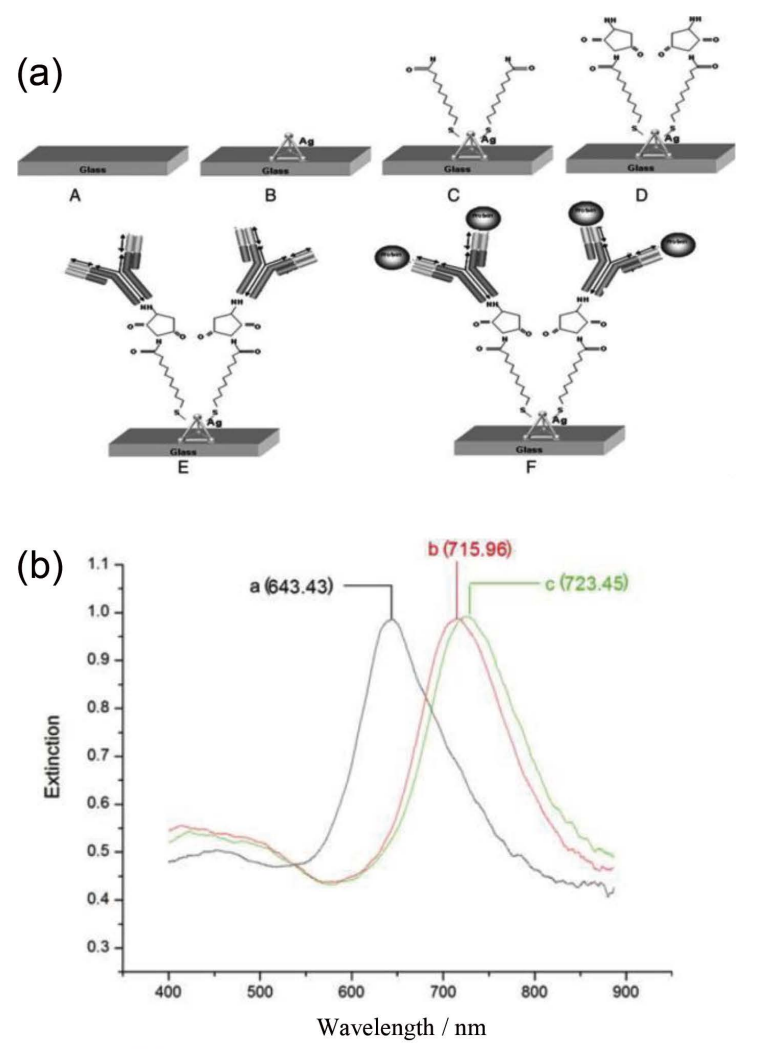

Fig. 8 (a) Schematic of preparation of LSPR sensor chips for albumin detection. (A - E) Stepwise deposition and functionalization of AgNPs and (F) albumin immunoassay. (b) LSPR spectra of a) AgNPs, b) functionalized AgNPs, and c) albumin detection. Copyright $\odot 2010$ Institute of Biochemistry and Cell Biology, SIBS, $\mathrm{CAS}^{43}$

indicate that protein is leaking through the vascular membranes of kidneys..$^{43,44}$ The standard methods for precisely determining the amount of albumin in urine are immunoassay methods, including microfluidic immunoassay. ${ }^{45-49}$ Recently, colorimetric sensing platforms based on plasmonic materials have been used to quantify urinary albumin. ${ }^{43,50,51}$ These platforms are inexpensive, have a convenient sensor array, and can be further developed into portable devices for POC diagnosis.

In one study, colloidal AgNP solutions were used for the detection of microalbuminuria in urine. ${ }^{43}$ AgNPs were deposited on a glass slide by an evaporation technique, and then functionalized by 11-mercaptoundecanoic acid to form a selfassembled monolayer (SAM) on the AgNP surfaces. The SAMfunctionalized AgNPs were consecutively functionalized by EDC/NHS and anti-albumin antibody. Schematic of preparation of LSPR sensor chips for albumin detection is shown in Fig. 8(a). Since this technique was based on immunoassay, the assay provided high selectivity and specificity to albumin. A series of albumin solutions with concentrations ranging from $1 \mathrm{ng} \mathrm{mL} \mathrm{m}^{-1}-1 \mu \mathrm{g} \mathrm{mL}^{-1}$ were detected. The sensor chips were incubated at different concentrations of albumin $30 \mathrm{~min}$ prior to recording the LSPR spectra. When the albumin concentration was increased, a blueshift in the LSPR peak of AgNPs was observed (Fig. 8(b)). Under optimum conditions, this technique led to a wide concentration range with a detection limit of $1 \mathrm{ng} \mathrm{mL} \mathrm{m}^{-1}$. Furthermore, this technique can be used to determine the albuminuria of a patient within a short assay time without sample purification. 
(a)

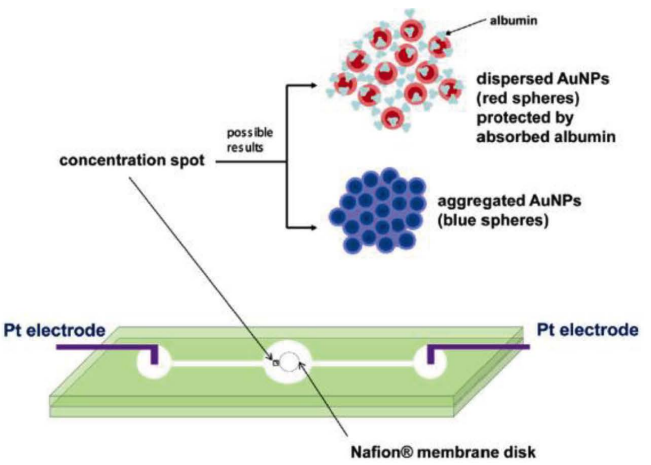

(b)

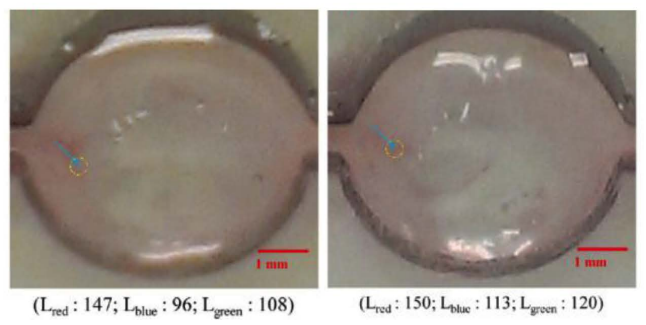

(c)

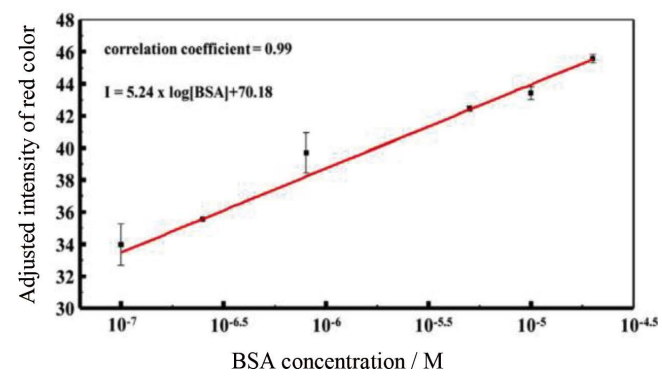

Fig. 9 (a) Schematic of a microconcentration device for albumin detection. The upper inset shows the albumin sensing concept of this device. The color of the concentrated AuNPs remains red when the particles are dispersed due to the steric hindrance of the absorbed albumin and changes to blue when the particles are aggregated. (b) Photographic images of concentrated AuNPs in artificial urine with spiked albumin at concentrations of 20 (left) and $0.1 \mu \mathrm{M}$ (right). (c) Linear relation between the adjusted intensities of the red chromatic values and albumin concentrations ranging from 0.1 to $20 \mu \mathrm{M}$. Copyright $\odot 2017$ Elsevier B.V..$^{50}$

An AuNP-based colorimetric method using a membrane disk was also developed for the determination of albumin content in artificial urine. ${ }^{50}$ Figure 9 (a) shows a schematic of the microconcentration device containing a Nafion ${ }^{\circledR}$ membrane disk. The upper plastic plate contains the center reservoir connected to two side reservoirs through channels. An electrode is immersed in each of the two side reservoirs for the application of an external electric field. The upper inset of this figure shows the concept of albumin sensing using this device. Under alkaline conditions, when the AuNP solution was spiked with albumin, the anionic albumin hindered the concentrated AuNPs from aggregating and their color remained red. Without steric hindrance of the absorbed albumin, AuNPs aggregated and the color turned to blue. The color change of the concentrated AuNPs caused by the albumin concentration was monitored using a camera. Figure 9(b) shows typical photographic images of the concentrated AuNPs in artificial urine with spiked albumin at concentrations of 20 (left) and $0.1 \mu \mathrm{M}$ (right). The chromatic values of red, green, and blue at the most colorcondensed locations, where the arrows point within the circle (a)

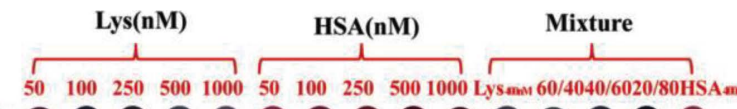
15m

(b)

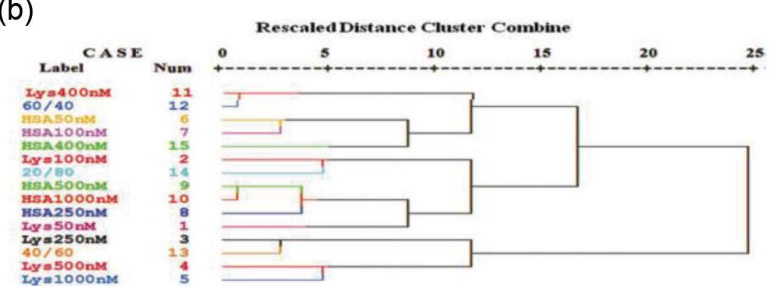

Fig. 10 (a) Color map for pure Lys and HSA at various concentrations and their mixtures spiked in human urine. (b) HCA for the corrected $\mathrm{R}, \mathrm{G}$, and $\mathrm{B}$ values on the sensor array. Copyright $\odot 2017$ The Royal Society of Chemistry. ${ }^{51}$

areas (Fig. 9(b)), were measured. Plots of the adjusted intensities of the red chromatic values versus albumin concentrations ranging from $0.1-20 \mu \mathrm{M}$ and a corresponding linear fit are shown in Fig. 9(c).

In one study, a colorimetric probe using AuNPs was developed for detecting multiple proteins. $^{51}$ Using five different concentrations of $\mathrm{NaCl}$ solutions, AuNP colloidal solutions exhibited different aggregation behaviors for different proteins due to their different ionic strengths, leading to a diverse color change. In this system, the target protein was first mixed with AuNPs, and then, different concentrations of $\mathrm{NaCl}$ solution were added to the mixture. As the AuNPs would exhibit different aggregation behaviors under different ionic strengths, as well as in the presence of different proteins, colorimetric patterns were obtained from each sample. The observable patterns were recorded using a smartphone camera, and the digital images were used for the investigation. This sensing system could not only discriminate 12 types of protein but also discriminate pure protein and the mixture of proteins in urine. Lysine (Lys) and human serum albumin (HSA) at various concentrations, as well as their mixture in urine samples, could further be distinguished through smartphone image acquisition and the colorimetric sensor array image could be further processed using hierarchical cluster analysis (HCA). The color map for pure Lys and HSA at various concentrations and their mixtures spiked in human urine and HCA for the corrected R, $\mathrm{G}$, and $\mathrm{B}$ values on the sensor array are shown in Fig. 10 .

MNPs have also been used for urinary protein detection using the SERS technique. ${ }^{52}$ A molecularly imprinted polymer (MIP)based plasmonic immunosandwich assay was developed for glycoprotein detection. A gold-based boronate affinity MIP array was used to specifically extract the target glycoprotein from complex samples. The extracted glycoprotein was then labeled with boronate affinity silver-based Raman nanotags. For sandwich-like complexes, the surface plasmon wave generated from the plasmonic nanoparticles enhanced the SERS signal of glycoprotein. The glycoprotein detection was achieved in solutions down to $2.9 \times 10^{-14} \mathrm{M}$. This technique was further employed for detecting glycoprotein in human urine. More recently, an SERS technique using AuNPs trapped in the nanochannels of a BRDVD has been used for albumin 
detection. ${ }^{40}$ A Raman peak at $1208 \mathrm{~cm}^{-1}$ corresponding to the $\mathrm{C}-\mathrm{N}$ stretching mode of albumin was detected from the mixture of artificial urine and albumin solutions. The minimum concentration of albumin that could be measured using this technique was $0.1 \mu \mathrm{g} \mathrm{mL}^{-1}$. Given the specific molecular information provided by Raman spectroscopy, this technique can be used clinically for albumin detection well below the normal concentration range of urinary albumin.

Another interesting point regarding urinary protein is its interference in creatinine detection. Given a high protein concentration in urine, it can be difficult to detect creatinine using colorimetric-probe-based AuNPs because the protein hinders the AuNP aggregation. Recently, we have developed a colorimetric probe using an AuNP colloidal solution to detect creatinine in the urine of proteinuria patients. ${ }^{53}$ Color changes in the AuNP colloidal solution in the presence of varying concentrations of creatinine and HSA showed different features and could be detected using a hyperspectral imaging technique and a smartphone camera. The relevant concentration ranges of creatinine and HSA were 5-200 and 50-250 mg dL ${ }^{-1}$, respectively. Contour plots of the red and blue chromaticity levels were obtained using color mapping of the mixture solutions containing AuNPs with creatinine and HSA. Twodimensional fitting equations obtained from the contour plots were adopted to determine the creatinine concentration in the urine of proteinuria patients. This practical technique can be used for rapid screening, and further developed as a household biosensing device for urinalysis.

\subsection{Glucose}

Normally, the blood glucose level is used as a clinical indicator of diabetes. Nevertheless, it is inconvenient and painful to draw blood by hand pricking, and this procedure may lead to infection. In contrast, the use of urine glucose is much simpler and safer. In addition, the presence of glucose in urine is a more direct indicator of serious cases of diabetes. When the glucose concentration in urine ranges $500-1000 \mathrm{mg} \mathrm{L}^{-1}(2.8-5.6 \mathrm{mM})$, the urine test is positive. ${ }^{54} \mathrm{~A}$ colorimetric assay for urinary glucose detection has attracted much attention because of its low cost, visuality, and potential for on-site detection. Although several colorimetric glucose sensors have achieved satisfactory sensitivity, their real practicability is limited by the requirement of complex sample pretreatment, such as centrifugal separation. ${ }^{55-58}$

Radhakumary and Sreenivasan undertook an impactful study on the colorimetric method using AuNPs for urinary glucose detection. ${ }^{59}$ To enable AuNPs for glucose detection, they were functionalized with the thiol chemical of 16-mercaptohexadecanoic acid (16-MHDA) and then glucose oxidase (GOD) using carbodiimide chemistry. The GODfunctionalized AuNPs was with selectivity toward glucose. A color change of the GOD-functionalized AuNPs from red to blue was observed upon interaction with glucose. When the carboxylic group (-COOH) of 16-MHDA was conjugated with the amino group of GOD, the LSPR peak showed a redshift. When glucose solutions in a concentration range of $10-$ $100 \mu \mathrm{g} \mathrm{mL}^{-1}$ were added, the LSPR peaks were shifted from $535-569 \mathrm{~nm}$, indicating AuNP aggregation. The reaction was instant, and no incubation time was required between glucose and GOD-functionalized AuNPs. LSPR spectra of the GODfunctionalized AuNPs in the presence of glucose at various concentrations from $10-100 \mathrm{~g} \mathrm{~mL}^{-1}$ are shown in Fig. 11(a). To determine the capability of this method for urinary glucose detection, glucose solution was spiked into the urine sample, which was then mixed with GOD-functionalized AuNPs. The LSPR peak redshift for $21 \mathrm{~nm}$ is shown in Fig. 11(b).
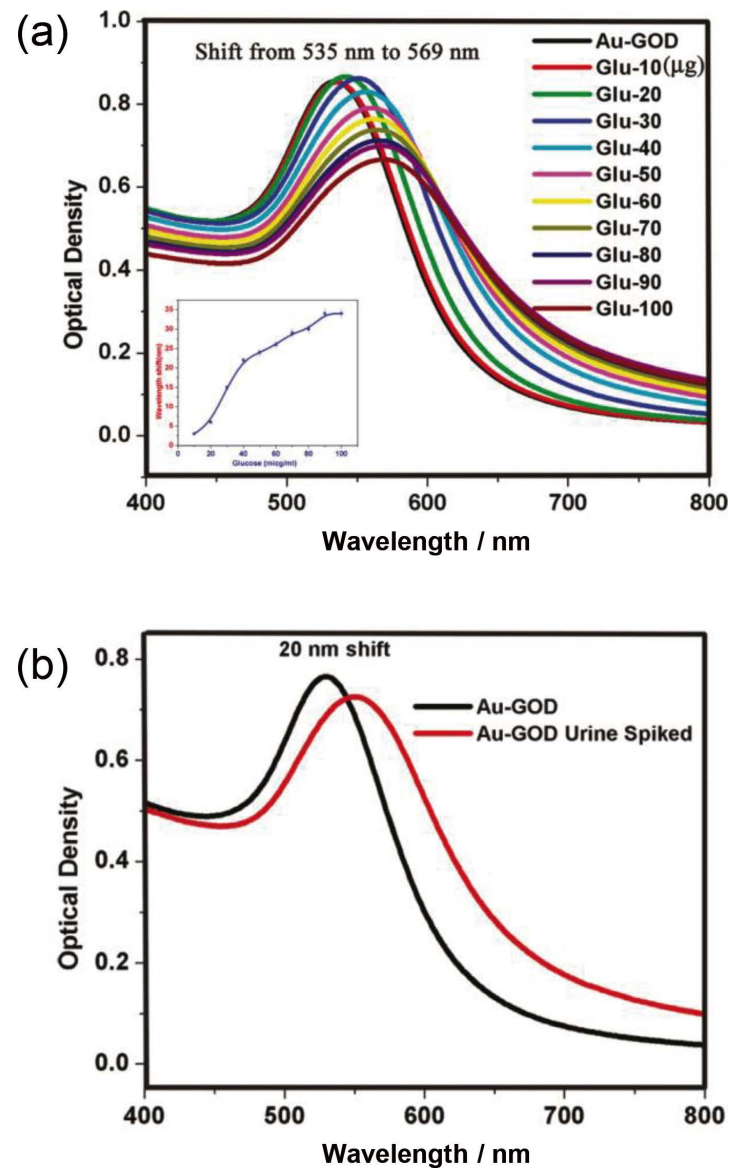

Fig. 11 (a) LSPR spectra of GOD-functionalized AuNPs, revealing redshift upon reactions with different quantities of glucose at various concentrations of $10-100 \mu \mathrm{g} \mathrm{mL}^{-1}$. The inset of (a) shows the relation between the wavelength shift and the glucose concentration. (b) LSPR spectra of GOD-functionalized AuNPs (black line) and GODfunctionalized AuNPs with $50 \mu \mathrm{g} \mathrm{mL}^{-1}$ glucose spiked into the urine sample. Copyright@2011 American Chemical Society. ${ }^{59}$

Recently, gold nanorods (GNRs) have also been used for urine glucose detection. ${ }^{55}$ Enzymatic reaction-mediated etching of GNRs was developed for on-site detection of urine glucose. With the catalysis of $\mathrm{MoO}_{4}{ }^{2-}$, GNRs were efficiently etched by $\mathrm{H}_{2} \mathrm{O}_{2}$, which was generated by the glucose-GOD enzymatic reaction. An evident blueshift of the LSPR peak of the GNRs was observed by a UV-vis spectrometer. Furthermore, a color change of the GNR solution from blue to red was easily observed by the naked eye. Under optimal conditions, an excellent sensitivity toward glucose was obtained with a detection limit of $0.1 \mu \mathrm{M}$. The visual detection of urine glucose in the urine sample revealed the applicability of this method for determining the health status of diabetes patients. Figure 12(a) shows a photographic image of colloidal GNR solutions mixed with different concentrations of glucose, revealing colorimetric changes of up to a $30-\mu \mathrm{M}$ glucose concentration. The change in the LSPR spectra of the colloidal GNR solutions in response to an increasing glucose concentration and plots depicting the relation between $\Delta \lambda$ the of GNRs and glucose concentrations ranging from $0.3-30 \mathrm{M}$ are shown in Figs. 12(b) and 12(c), respectively. A 500-fold dilution of the original sample was prepared using a buffer solution. Different glucose concentrations were spiked into healthy human urine, and the color responses of GNRs to different concentrations of glucose were observed 
(a)

(b)
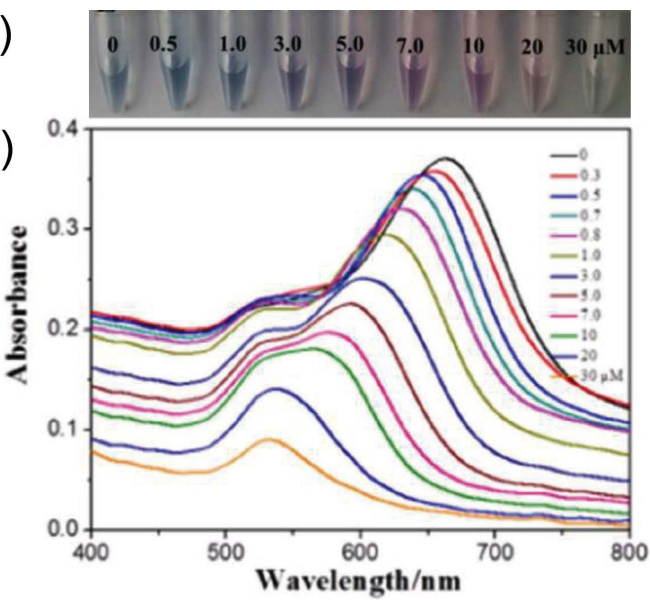

(C)

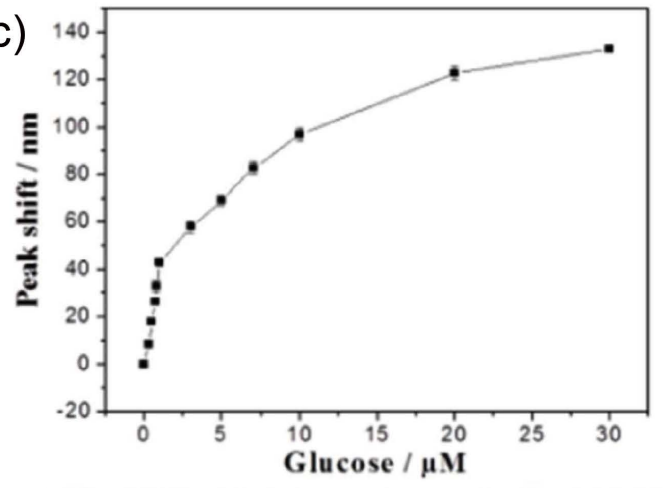

(d)
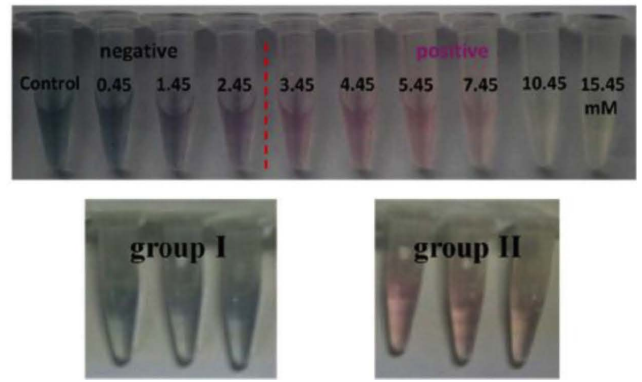

Fig. 12 (a) Photographic image of colloidal GNR solutions mixed with different concentrations of glucose. (b) Change in LSPR spectra of colloidal GNR solutions in response to glucose concentration. (c) Plots depicting the relation between the $\Delta \lambda$ of GNRs and the glucose concentration. (d) Photographic images of different concentrations of glucose spiked into healthy human urine (top) and a comparison of urine test results among three healthy persons (group I) and three diabetes patients (group II). Copyright@2017 Elsevier B.V. ${ }^{55}$

(Fig. 12(d), top). When the glucose concentration in the urine was higher than $2.8 \mathrm{mM}$, the detection result was considered to be positive for diabetes. Urine samples from three healthy volunteers and three diabetes volunteers were tested. Comparing the results with the standard color (Fig. 12(d), top), diabetes patients could be distinguished from healthy individuals. The photographic images of GNRs in the case of healthy individuals (Fig. 12(d), group I) and diabetes patients (Fig. 12(d), group II) are shown in Fig. 12(d). This confirms that this method can be used for detecting glucose in urine for diabetes diagnosis.

An alternative colorimetric probe for urine glucose detection is an oxidase-like activity method. ${ }^{56-58}$ The visible color observable based on this method is not due to LSPR. Nevertheless, this method takes advantage of the catalytic activity of palladium nanoparticles to enhance the oxidation reaction, leading to a color change in the analyzed solution. The color change caused by this method can be observed by the naked eye and quantified by a spectrometer. The detection of urine glucose using this method was achieved for a glucose concentration range of $50-100 \mathrm{mg} \mathrm{dL}^{-1}$, making it practically applicable.

\subsection{Other chemicals in urine}

The colorimetric method based on the LSPR of MNPs can also be used to detect other chemicals in urine, such as catecholamine neurotransmitters, ${ }^{60}$ thymine, ${ }^{61}$ medicines (e.g. cimetidine), ${ }^{62}$ and biothiols. ${ }^{63}$ A high-performance colorimetric sensor array has been developed for the detection of catecholamines including neurotransmitters, such as dopamine, epinephrine, and norepinephrine based on reducing the ability for silver deposition on the GNR surface. ${ }^{60}$ The blueshift of the longitudinal LSPR peak of GNRs creates a unique pattern for each neurotransmitter. Distinct patterns correlated with each neurotransmitter were collected by recording the absorbance spectra of GNRs at different concentrations. A color map and chemometric methods, including HCA, principal component analysis, and linear discrimination analysis, were employed to analyze the array responses. The detection limits of this technique for dopamine, epinephrine, and norepinephrine were 5.0, 1.0, and $1.0 \mu \mathrm{g} \mathrm{mL}^{-1}$, respectively. The proposed colorimetric array could be used to differentiate individual neurotransmitters as well as their mixtures in urine samples.

The qualitative and quantitative determination of thymine in a DNA standard and urine samples have also been investigated using AuNPs as a label-free colorimetric sensor. ${ }^{61}$ The mechanism for thymine detection was demonstrated through the color change of AuNPs from pink to blue, caused by the shift of the LSPR peak. The linear range obtained for the quantitative determination of thymine was found to be $10-1200 \mathrm{ng} \mathrm{mL}^{-1}$ with a detection limit of $3 \mathrm{ng} \mathrm{mL}^{-1}$. This technique can be used to detect thymine in urine in a concentration range of 46.6 $101.2 \mathrm{ng} \mathrm{mL}^{-1}$. The accuracy of this method was calculated by spiking thymine into urine samples, leading to recovery rates in the range of $93.5-96.3 \%$.

A colorimetric sensor for cimetidine detection based on D-xylose-hindered AuNPs has also been developed. ${ }^{62}$ Cimetidine is a stomach acid reducer used to treat and prevent certain types of stomach ulcers. It acts primarily on $\mathrm{H}-2$ receptors on parietal cells, competitively inhibiting the action of histamine and gastric acid secretion. Cimetidine causes AuNP aggregation due to the formation of a strong covalent Au-N bond. When the cimetidine concentration increased, the LSPR peak was found to shift from $533-680 \mathrm{~nm}$ and the color of the solutions changed from red to blue due to AuNP aggregation. This method exhibited a wide detection range with low detection limits of $1.0 \times 10^{-8} \mathrm{M}$ in water and $3.0 \times 10^{-8} \mathrm{M}$ in human urine.

\section{Portable Platforms Based on Colorimetric Probes Using LSPR for Human Health Monitoring}

The development of a diagnostic device for POC application is one of the most challenging objectives in the clinical field. This paper provides information regarding portable devices based on colorimetric methods, while focusing on those based on the LSPR of MNPs for urine detection. ${ }^{11,50,64-69}$ Portable-platformbased colorimetric probes can also be used for immunosensing systems, such as those used for cancer detection ${ }^{64}$ and sepsis diagnosis. ${ }^{65}$ This platform can also be very useful for health 
monitoring based on the detection of compounds in biomolecules produced from the human body, such as lactate in oral fluid and sweat ${ }^{66}$ salivary cortisol, ${ }^{67}$ chloride in sweat, ${ }^{68}$ albumin, ${ }^{50}$ glucose, ${ }^{11}$ and uric acid. ${ }^{69}$

The 3D-printing technology is a powerful tool for developing several portable sensing platforms, including colorimetric probes for biosensor applications..$^{11,66,70}$ A disposable minicartridge was printed by a 3D printer to detect chemiluminescence derived from the enzyme-coupled reaction of lactate in oral fluid and sweat. ${ }^{66}$ Furthermore, lactate oxidase was coupled with horseradish peroxidase for lactate detection. The imaging signals and data evaluations were performed using a smartphone. With this technique, lactate levels could be quantified within $5 \mathrm{~min}$. The detection limits of this technique for oral fluid and sweat were 0.5 and $0.1 \mathrm{mM}$, respectively. Recently, chloride in sweat, which is a diagnostic marker for cystic fibrosis, has been monitored by a smartphone-based fluorescence sensor. ${ }^{68}$ This technique could detect a wide range of chloride concentrations $(0.8-200.0 \mathrm{mM})$ covering the concentration range necessary for primary diagnosis of cystic fibrosis. A method for colorimetric detection by an immunosensor based on a sandwich strategy, which can be performed by a smartphone camera for the detection of cancer antigen 125 (CA125), was also developed. ${ }^{64}$ This showed promising results for a screening test of CA125 in human serum. Portable-platform-based colorimetric detection using smartphones is a very attractive possibility for this purpose. However, there are not many colorimetric detections for urine using smartphones.

The following studies were conducted on portable platforms based on colorimetric detection for diagnosing the chemicals in urine. In one study, a microconcentrator chip was fabricated using a Nafion ${ }^{\circledR}$ cation-exchange membrane and a camera was used to acquire the images of AuNPs and AgNPs with albumin from the sensor chips. ${ }^{50}$ An external electric field was applied to induce AuNP aggregation. When the electric field was applied, the color of the AuNPs changed from red to blue. With albumin, the AuNP aggregation was hindered even under an applied electric field. As the color of AuNPs in the sensor chip changed depending on the albumin concentration, the red chromatic value obtained from the digital image was used for evaluating the albumin concentration. A linear correlation between the intensity of the red chromatic value and the logarithm value of the albumin concentration was found in the range of $0.1-20.0 \mu \mathrm{M}$. We also proposed image acquisition from silver nanoprisms (AgNPrs) using a combination of transmitted and scattered light for glucose detection. ${ }^{11}$ A smartphone camera assembled with a 3D-printed chamber was developed and used for recording transmitted and scattering images of a solution prior to calculation of empirical absorption. Schematics of the device and transmitted and scattering images acquisitions are shown in Fig. 13(a). A suitable linearity between the signal and glucose concentration was observed in the concentration range of $20-100 \mu \mathrm{M}$, and $R^{2}$ and a detection limit of 0.9869 and $19.8 \mu \mathrm{M}$ were obtained, respectively. Transmitted and scattering images of colloidal solutions of AgNPrs with glucose at various concentrations and the plots of the optical signals as a function of glucose concentration are shown in Fig. 13(b). This system demonstrates great potential for further development as a portable device for glucose detection in urine for diabetes screening.

As has been discussed, one major advantage of using urine over blood is the ease of sampling, which generally confers no disturbance to patient integrity, unlike needle-based extraction. Although the obtained data do not provide exact results for diagnosis, unlike the results obtained from blood samples, (a)
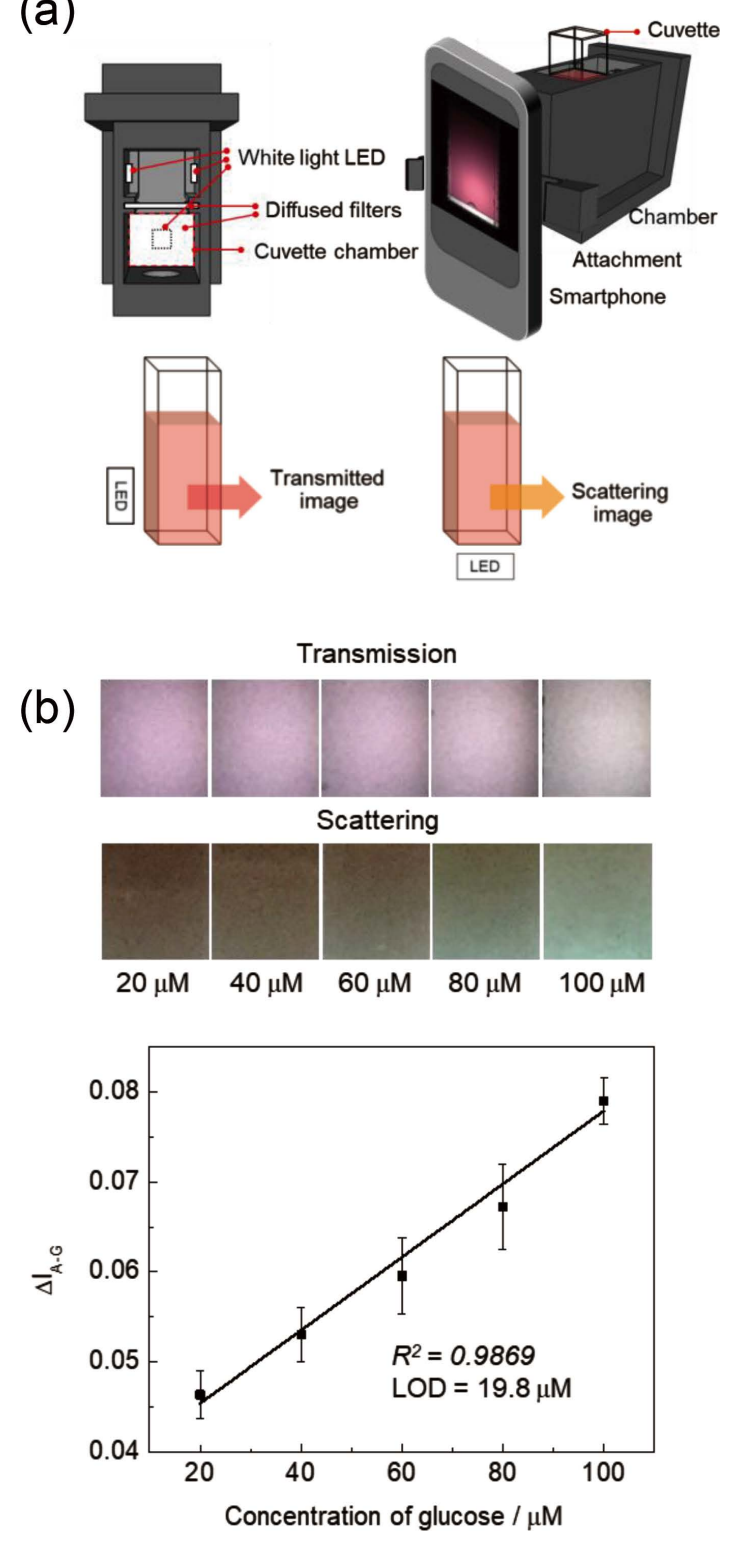

Fig. 13 (a) 3D-printed device for recording transmitted and scattering images of colloidal solution of AgNPrs. (b) Transmitted and scattering images of colloidal solutions of AgNPrs with glucose at various concentrations from 20 to $100 \mu \mathrm{M}$ and the plots of the optical signals as a function of glucose concentration. Copyright@2019 Japan Society for Analytical Chemistry. ${ }^{11}$

detection using urine is a useful technique for rapid health monitoring and screening. Furthermore, health monitoring using urine samples can be integrated into a smart toilet system,${ }^{71}$ which is a convenient method for individuals to check their own health status at home.

\section{Conclusions}

Colorimetric methods based on LSPR provide simple, rapid, real-time, nonlabelled, and sensitive detection of chemical components in urine. These methods can be used for detecting creatinine, albumin, and glucose in human urine and can be extended to detect some specific chemicals that might exist in human urine, such as catecholamine neurotransmitters, thymine, 
and various medicines. The colorimetric probes used to detect these biochemicals in human urine have shown excellen detection with high sensitivity, selectivity, and recovery rates. According to all the above mentioned merits, colorimetric probes based on LSPR are fit for use as rapid screening techniques for the diagnosis of diseases, and can very likely be used as household biosensors for personal health monitoring.

\section{Acknowledgements}

This research was supported by Adaptable and Seamless Technology Transfer Program Through Target-driven R\&D (A-STEP) of the Japan Science and Technology Agency (JST) (VP30118067841), and the Japan Society for the Promotion of Science (JSPS) KAKENHI Grant Number JP20K21140.

\section{References}

1. K. L. Kelly, E. Coronado, L. L. Zhao, and G. C. Schatz, J. Phys. Chem. B, 2003, 107, 668.

2. M. M. Miller and A. A. Lazarides, J. Phys. Chem. B, 2005, 109, 21556.

3. J. E. Millstione, S. J. Hurst, G. S. M'etraux, J. I. Cutler, and C. A. Mirkin, Small, 2009, 5, 646

4. K. M. Mayer and J. H. Hafner, Chem. Rev., 2011, 111, 3828.

5. O. Kvítek, J. Siegel, V. Hnatowicz, and V. Švorčík, J. Nanomater, 2013, 2013, 743684.

6. K. Ueno, S. Juodkazis, M. Mino, V. Mizeikis, and H. Misawa, J. Phys. Chem. C, 2007, 111, 4180.

7. S. Zheng, K. Yong, I. Roy, X. Dinh, X. Yu, and F. Luan, Plasmonics, 2011, 6, 491.

8. J. Sun, Y. Xianyu, and X. Jiang, Chem. Soc. Rev., 2014, 43, 6239.

9. N. Elahi, M. Kamali, and M. H. Baghersad, Talanta, 2018, 184,537

10. Y. Yano, M. Nisougi, Y. Yano-Ozawa, T. Ohguni, A. Ogawa, M. Maeda, T. Asahi, and T. Zako, Anal. Sci., 2019, 35, 685.

11. C. Lertvachirapaiboon, T. Maruyama, A. Baba, S. Ekgasit, K. Shinbo, and K. Kato, Anal. Sci., 2019, 35, 271.

12. P. Kumar, R. Jaiwal, and C. S. Pundir, Anal. Biochem., 2017, 537, 41.

13. U. Lad, S. Khokhar, and G. M. Kale, Anal. Chem., 2008, 80, 7910.

14. M. J. Ruedas-Rama and E. A. H. Hall, Anal. Chem., 2010, 82,9043

15. U. Jain, J. Narang, and N. Chauhan, Int. J. Adv. Res., 2015, 3,1482

16. C. S. Pundir, P. Kumar, and R. Jaiwal, Biosens. Bioelectron., 2019, 126, 707.

17. R. Cánovas, M. Cuartero, and G. A. Crespo, Biosens. Bioelectron., 2019, 130, 110.

18. M. Li, Y. Du, F. Zhao, J. Zeng, C. Mohan, and W. Shih, Biomed. Opt. Express, 2015, 6, 849.

19. E. Liotta, R. Gottardo, L. Bonizzato, J. P. Pascali, A. Bertaso, and F. Tagliaro, Clin. Chim. Acta, 2009, 409, 52.

20. S. Yadav, R. Devi, P. Bhar, S. Singhla, and C. S. Pundir, Enzyme Microbiol. Technol., 2012, 50, 247.

21. C. Nieh, S. Tsujimura, O. Shirai, and K. Kano, Anal. Chim. Acta, 2013, 767, 128

22. T. T. Zava, S. Kapur, and D. T. Zava, Anal. Chim. Acta, 2013, 764, 64

23. Y. He, X. Zhang, and H. Yu, Microchim. Acta, 2015, 182,
2037.

24. J. Sittiwong and F. Unod, Spectrochim. Acta, Part A, 2015, 138,381

25. J. Du, B. Zhu, W. R. Leow, S. Chen, T. C. Sum, X. Peng, and X. Chen, Small, 2015, 11, 4104.

26. H. Du, R. Chen, J. Du, J. Fan, and X. Peng, Ind. Eng. Chem. Res., 2016, 55, 12334.

27. S. Mohammadi and G. Khayatian, Microchim. Acta, 2015 , 182, 1379.

28. M. T. Alula, L. Karamchand, N. R. Hendricks, and J. M. Blackburn, Anal. Chim. Acta, 2018, 1007, 40.

29. E. P. Randviir, D. K. Kampouris, and C. E. Banks, Analyst, 2013, 138,6565 .

30. H. Cui, W. Wang, C. F. Duan, Y. P. Dong, and J. Z. Guo, Chem. Eur. J., 2007, 13, 6975.

31. H. Chi, B. Liu, G. Guan, Z. Zhang, and M. Han, Analyst, 2010, 135, 1070 .

32. Y. He and R. Peng, Nanotechnology, 2014, 25, 455502.

33. D. I. Gittins and F. Caruso, Angew. Chem. Int. Ed., 2001, 40, 3001 .

34. T. Sismanoglu, S. Pura, and A. S. Bastug, Rev. Inorg. Chem., 2003, 23, 25.

35. Y. Q. Wen, F. F. Xing, S. J. He, S. P. Song, L. H. Wang, Y. T. Long, D. Li, and C. H. Fan, Chem. Commun., 2010, 46, 2596.

36. A. M. E. Badawy, T. P. Luxton, R. G. Silva, K. G. Scheckel, M. T. Suidan, and T. M. Tolaymat, Environ. Sci. Technol., 2010, 44, 1260.

37. M. S. Frost, M. J. Dempsey, and D. E. Whitehead, Colloid. Surf. Physicochem. Eng. Aspect., 2017, 518, 15.

38. A. R. Butler and C. Glidewell, J. Chem. Soc. Perkin Trans. II, 1985, 9, 1465.

39. P. G. Sutariya, A. Pandya, A. Lodha, and S. K. Menon, Talanta, 2016, 147, 590.

40. N. Chamuah, A. Saikia, A. M. Joseph, and P. Nath, Sens. Actuators, B, 2019, 285, 108.

41. J. Sittiwong and F. Unob, Anal. Sci., 2016, 32, 639.

42. R. Rajamanikandan and M. Ilanchelian, Anal. Methods, 2018, 10, 3666.

43. T. Lai, Q. Hou, H. Yang, X. Luo, and M. Xi, Acta Biochim. Biophys. Sin., 2010, 42, 787.

44. C. Lin, C. Tseng, T. Chuang, D. Lee, and G. Lee, Analyst, 2011, 136, 2669.

45. R. J. Stamp, Ann. Clin. Biochem., 1988, 25, 442.

46. T. L. Wu, P. Y. Chang, C. C. Li, K. C. Tsao, C. F. Sun, and J. T. Wu, Ann. Clin. Lab. Sci., 2005, 35, 149.

47. M. Lu, F. Ibraimi, D. Kriz, and K. Kriz, Biosens. Bioelectron., 2006, 21, 2248.

48. K. M. Park, S. K. Lee, Y. S. Sohn, and S. Y. Choi, Electron Lett., 2008, 44, 185.

49. W. Laiwattanapaisal, T. Songjaroen, T. Maturos, T. Lomas, A. Sappat, and A. Tuantranont, Sensors, 2009, 9, 10066.

50. T. Lai, T. Chang, and S. Wang, Sens. Actuators, B, 2017, 239, 9.

51. F. Wang, Y. Lu, J. Yang, Y. Chen, W. Jing, L. He, and Y. Liu, Analyst, 2017, 142, 3177.

52. X. Tu, P. Muhammad, J. Liu, Y. Ma, S. Wang, D. Yin, and Z. Liu, Anal. Chem., 2016, 88, 12370.

53. C. Lertvachirapaiboon, A. Baba, K. Shinbo, and K. Kato, Anal. Sci., 2020, 36, 1495.

54. T. Urakami, J. Suzuki, A. Yoshida, H. Saito, and H. Mugishima, Diabetes Res. Clin. Pract., 2008, 80, 473.

55. Z. Zhang, Z. Chen, F. Cheng, Y Zhang, and L. Chen, Biosens. Bioelectron., 2017, 89, 932.

56. L. Su, J. Feng, X. Zhou, C. Ren, H. Li, and X. Chen, Anal. 
Chem., 2012, 84, 5753.

57. L. Su, W. Qin, H. Zhang, Z. U. Rahman, C. Ren, S. Ma, and X. Chen, Biosens. Bioelectron., 2015, 63, 384.

58. X. Zheng, Q. Zhu, H. Song, X. Zhao, T. Yi, H. Chen, and X. Chen, ACS Appl. Mater. Interfaces, 2015, 7, 3480.

59. C. Radhakumary and K. Sreenivasan, Anal. Chem., 2011, 83, 2829.

60. S. Jafarinejad, M. Ghazi-Khansari, F. Ghasemi, P. Sasanpour, and M. R. Hormozi-Nezhad, Sci. Rep., 2017, 7, 8266.

61. K. Shrivas, N. Nirmalkar, S. S. Thakur, R. Kurrey, D. Sinha, and R. Shankar, RSC Adv., 2018, 8, 24328.

62. F. Hu, P. Wu, R. Wang, W. Liu, and H. He, Analyst, 2018 143, 2369.

63. D. Yuan, J. J. Liu, H. H. Yan, C. M. Li, C. Z. Huang, and J. Wang, Talanta, 2019, 203, 220.
64. O. Hosu, A. Ravalli, G. M. L. Piccolo, C. Cristea, R. Sandulescu, and G. Marrazza, Talanta, 2017, 166, 234.

65. S. Kim, I. Cho, G. Lim, G. Park, and S. Paek, Biosens. Bioelectron., 2017, 98, 7.

66. A. Roda, M. Guardigli, D. Calabria, M. M. Calabretta, L. Cevenini, and E. Michelini, Analyst, 2014, 139, 6494.

67. M. Zangheri, L. Cevenini, L. Anfossi, C. Baggiani, P. Simoni, F. D. Nardo, and A. Roda, Biosens. Bioelectron. 2015, 64, 63.

68. C. Zhang, J. P. Kim, M. Creer, J. Yang, and Z. Liu, Biosens. Bioelectron., 2017, 97, 164.

69. M. Ali, M. A. U. Khalid, I. Shah, S. W. Kim, Y. S. Kim, J. H. Lim, and K. H. Choi, New J. Chem., 2019, 43, 7636.

70. S. Kanchi, M. I. Sabela, P. S. Mdluli, Inamuddin, and K. Bisetty, Biosens. Bioelectron., 2018, 102, 136.

71. J. Bae and H. Lee, IEEE Access, 2018, 6, 78751. 